\title{
Principal Submatrices V: Some Results Concerning Principal Submatrices of Arbitrary Matrices*
}

\author{
R. C. Thompson**
}

(April 3, 1968)

\begin{abstract}
This paper studies: (i) interlacing properties for the real eigenvalues of matrices; (ii) symmetric matrices with many equal principal minors; (iii) the determinantal characterization of the rank of a matrix.
\end{abstract}

Key Words: Eigenvalues, matrix, principal submatrices, rank, symmetric matrix.

\section{Introduction}

This paper is the fifth $[1,2,3,4]^{1}$ in a continuing series of papers in which the totality of the principal submatrices of a matrix are studied. In the first paper [1] in this series, a large number of inequalities were established linking the eigenvalues of all of the principal $(n-1) \times(n-1)$ submatrices of an $n \times n$ normal or Hermitian matrix $A$ to the eigenvalues of $A$. In the second and third paper [2,3] in the series, the cases of equality were found when $A$ is Hermitian for some of the inequalities obtained in [1]. In [4] the amount of freedom with which one can specify the eigenvalues of the principal $(n-1) \times(n-1)$ submatrices of an $n \times n$ Hermitian matrix was examined.

In this paper, we establish a number of theorems concerning the behavior of the principal submatrices of not necessarily normal matrices. In section 3 of this paper, we show that a new type of interlacing theorem, that was proved in [2] for Hermitian matrices, remains valid for certain other classes of matrices, particularly certain oscillation matrices. We also show in section 3 that for arbitrary real matrices there is still valid a weak type of interlacing theorem (Theorem 2) which, when applied to a nonnegative matrix $A$, yields an interesting result (Theorem 3 ) concerning the maximal eigenvalues of the different principal submatrices of $A$. In section 4 we study certain classes of matrices for which, in the determinantal characterization of rank, it suffices to consider principal subdeterminants. Although this section is partly expository, extensions and new proofs of a number of known results are presented. We close the section with a new proof of the law of inertia. In section 5 we classify the symmetric matrices over an arbitrary field, for which all $r \times r$ principal subdeterminants are equal for three consecutive values of $r$.

\section{Notation}

Let $A$ be an $n \times n$ matrix. Let $\omega=\left\{i_{1}, i_{2}, \ldots, i_{r}\right\}$ and $\tau=\left\{j_{1}, j_{2}, \ldots, j_{s}\right\}$ be two sets of distinct positive integers, not exceeding $n$. Then $A[\omega \mid \tau]$ denotes the $r \times s$ submatrix of $A$ lying at the intersection of rows $i_{1}, \ldots, i_{r}$ and columns $j_{1}, \ldots ., j_{s}$. Usually $i_{1}, \ldots ., i_{r}$ and $j_{1}, \ldots ., j_{s}$ will be in increasing order. Let $Q_{n r}$ denote set of all such strictly increasing sequences $\omega=\left\{i_{1}, \ldots \ldots, i_{r}\right\}$. Let $f_{[\omega]}(\lambda)$ denote the characteristic polynomial of $A[\omega \mid \omega]$. Then we have the following fact:

$$
\sum_{\omega \in \mathbb{Q}_{n r}} f_{[\omega]}(\lambda)=((n-r) !)^{-1} f^{(n-r)}(\lambda)
$$

*The preparation of this paper was supported in part by the U.S. Air Force Office of Scientific Research, under Grant $698-67$.

**Present address, University of California, Department of Mathematics, Santa Barbara, Calif. 93106.

1 Figures in brackets indicate the literature references at the end of this paper. 
where $f^{(t)}(\lambda)$ denotes the $t$ th derivative of $f(\lambda)$. The formula (1) is well known and may be deduced by repeated differentiation of the determinant $\operatorname{det}(\lambda I-A)$, or by using the fact that the coefficients of the powers of $\mu$ in $\operatorname{det}(\mu I-(\lambda I-A))$ are sums of principal subdeterminants of $(\lambda I-A)$ (that is, sums over $Q_{n r}$ of the $\left.f_{[\omega]}(\lambda)\right)$ and by using Taylor's theorem to express

$$
\operatorname{det}(\mu I-(\lambda I-A))=\operatorname{det}((\mu-\lambda) I+A)=(-1)^{n} f(\lambda-\mu)
$$

as a polynomial in $\mu$.

When $k=n-1$ we let $i$ denote the unique positive integer $\leqslant n$ for which $i \notin \omega$. Then $f_{[\omega]}(\lambda)=f_{(i)}(\lambda)$ is the characteristic polynomial of $A(i \mid i)$, the principal submatrix of $A$ obtained by deleting row $i$ and column $i$ from $A$. In this case, (1) becomes

$$
\sum_{i=1}^{n} f_{(i)}(\lambda)=f^{\prime}(\lambda)
$$

The $n \times n$ matrix in which each entry is a one is denoted by $J_{n}$. We let superscript ${ }^{T}$ denote transpose. Finally, if matrix $A$ is $n \times n$, we say degree $A=n$.

\section{Interlacing Properties}

TheOREM 1. Suppose $\mathrm{n} \times \mathrm{n}$ matrix A possesses a real spectrum,

$$
\lambda_{1} \leqslant \lambda_{2} \leqslant \ldots \leqslant \lambda_{n}
$$

and that the spectrum

$$
\xi_{\mathrm{i} 1}, \xi_{\mathrm{i} 2}, \ldots ., \xi_{\mathrm{i}, \mathrm{n}-1}
$$

of the principal $(\mathrm{n}-1)$ square submatrix $\mathrm{A}(\mathrm{i} \mid \mathrm{i})$ of $\mathrm{A}$ is real for each $\mathrm{i}$, and for each $\mathrm{i}$ satisfies

$$
\lambda_{1} \leqslant \xi_{\mathrm{i} 1} \leqslant \lambda_{2} \leqslant \xi_{\mathrm{i} 2} \leqslant \ldots . \leqslant \lambda_{\mathrm{n}-1} \leqslant \xi_{\mathrm{i}, \mathrm{n}-1} \leqslant \lambda_{\mathrm{n}}
$$

Let $\xi_{1} \leqslant \xi_{2} \leqslant \ldots \xi_{\mathrm{n}-1}$ be the (necessarily real) roots of $\mathrm{f}^{\prime}(\lambda)$. Then, for fixed $\mathrm{j}, 1 \leqslant \mathrm{j} \leqslant \mathrm{n}-1$, either

(i) there is at least one i such that $\xi_{\mathrm{ij}}>\xi_{\mathrm{j}}$ and at least one i such that $\xi_{\mathrm{ij}}<\lambda_{\mathrm{j}}$; or

(ii) $\xi_{1 \mathrm{j}}=\xi_{2 \mathrm{j}}=\ldots .=\xi_{\mathrm{nj}}=\xi_{\mathrm{j}}$.

REMARK: It is known that Hermitian matrices, real symmetric matrices, and certain types of Jacobi and oscillation matrices, are examples of matrices satisfying the conditions of the theorem. For Hermitian and real symmetric matrices, Theorem 1 was proved in the paper [2] and heavily used in both [2] and [3].

Proof. If $\lambda_{j}=\lambda_{j+1}$ then, by the interlacing property (3), $\xi_{i j}=\lambda_{j}$ for each $i$. Moreover, as $\lambda_{j} \leqslant \xi_{j} \leqslant \lambda_{j+1}$, we also have $\xi_{j}=\lambda_{j}$. Thus $\xi_{i j}=\xi_{j}$ for all $i$, and hence (ii) is in force. So suppose $\lambda_{j}<\lambda_{j+1}$. In this case $\lambda_{j}<\xi_{j}<\lambda_{j+1}$. Assume, if possible, that $\xi_{i j} \geqslant \xi_{j}$ for all $i$, with strict inequality for at least one value of $i$. Then the roots of $f_{(\mathrm{i})}(\lambda)$ in the interval $\left(\xi_{j}, \infty\right)$ are $\xi_{i j}, \xi_{i, j+1}, \ldots ., \xi_{i, n-1}$. Thus $f_{(\mathrm{i})}\left(\xi_{j}\right)=0$ or $\operatorname{sign} f_{(\mathrm{i})}\left(\xi_{j}\right)=(-1)^{n-j}$, with the second possibility at least once. Thus, from (2), $f^{\prime}\left(\xi_{j}\right) \neq 0$. This is a contradiction. Consequently our hypothesis is untenable. Similarly one proves that $\xi_{i j} \leqslant \xi_{j}$ for all $i$, with strict inequality at least once, is impossible. Therefore, if $\xi_{i j}>\xi_{j}$ for at least one $i$ then $\xi_{i j}<\xi_{j}$ for at least one $i$, and conversely. This completes the proof.

We turn now to what we can say about arbitrary matrices.

Theorem 2. Let A be a matrix of real numbers. Suppose $\mathrm{f}^{(\mathrm{n}-\mathrm{k})}(\lambda)$ possesses real roots and let $\gamma_{\mathrm{k}}$ and $\delta_{\mathrm{k}}$ be, respectively, the largest and smallest real roots of $\mathrm{f}^{(\mathrm{n}-\mathrm{k})}(\lambda)$. Then, either

(i) for at least one $\omega \in \mathrm{Q}_{\mathrm{nk}}, \mathrm{A}[\omega \mid \omega]$ has a real eigenvalue $\alpha>\gamma_{\mathrm{k}}$; or 
(ii) for every $\omega \epsilon \mathrm{Q}_{\mathrm{nk}}, \gamma_{\mathrm{k}}$ is an eigenvalue of $\mathrm{A}[\omega \mid \omega]$. Furthermore, either

(iii) for at least one $\omega \epsilon \mathrm{Q}_{\mathrm{nk}}, \mathrm{A}[\omega \mid \omega]$ has a real eigenvalue $\beta<\delta_{\mathrm{k}}$; or

(iv) for every $\omega \epsilon \mathrm{Q}_{\mathrm{nk}}, \delta_{\mathrm{k}}$ is an eigenvalue of $\mathrm{A}[\omega \mid \omega]$.

Proof. Suppose, if possible, that no $f_{[\omega]}(\lambda)$ has a root in the interval $\left[\gamma_{k}, \infty\right)$. Then $f_{[\omega]}\left(\gamma_{k}\right)>0$ for each $\omega \epsilon Q_{n k}$. Since $f^{(n-k)}\left(\gamma_{k}\right)=0$, we have contradicted (1). So either some $f_{[\omega]}(\lambda)$ has a root in $\left(\gamma_{k}, \infty\right)$, or, if this is not true, then $f_{[\omega]}\left(\gamma_{k}\right) \geqslant 0$ for each $\omega$ and hence (by $\left.(1)\right), f_{[\omega]}(\gamma)=0$ for each $\omega$. This proves (i) and (ii). The proof of (iii) and (iv) is similar.

In the case of nonnegative matrices, Theorem 2 specializes to the following interesting fact.

THEOREM 3. Let A be a matrix of nonnegative numbers. Let $\rho_{\omega}$ denote the maximal eigenvalue of $\mathrm{A}[\omega \mid \omega] ; \omega \epsilon \mathrm{Q}_{\mathrm{nk}}$. Suppose $\mathrm{f}^{(\mathrm{n}-\mathrm{k})}(\lambda)$ has a real root and let $\gamma_{\mathrm{k}}$ be the maximal real root of $\mathrm{f}^{(\mathrm{n}-\mathrm{k})}(\lambda)$. Then, either

(i) for at least one $\omega \epsilon \mathrm{Q}_{\mathrm{nk}}, \rho_{\omega}>\gamma_{\mathrm{k}}$, or

(ii) for every $\omega \epsilon \mathrm{Q}_{\mathrm{nk}}, \rho_{\omega}=\gamma_{\mathrm{k}}$.

We note that case (ii) can occur for every $k$. An example of this situation is the matrix $J_{n}$. Each $k \times k$ principal submatrix of $J_{n}$ is $J_{k}$ and has $k$ as its maximal eigenvalue. The characteristic polynomial of $J_{n}$ is $f(\lambda)=\lambda^{n}-n \lambda^{n-1}$ so that $f^{(n-k)}(\lambda)$ has $k$ as its maximal root.

\section{On the Determinantal Characterization of Rank}

It is well known that the rank of a matrix $A$ is characterized as the degree of the largest nonvanishing subdeterminant of $A$, and it is also known that for certain classes of matrices it suffices to consider principal subdeterminants. In this section we study two quite general such classes which appear to cover all known classes of matrices possessing this property.

THEOREM 4. Let $\mathfrak{\wp}$ be an arbitrary field and let A be a matrix over $\mathfrak{\wp}$ such that the elementary divisors of A belonging to eigenvalue zero are all linear. Then rank A equals the degree of the largest nonvanishing principal subdeterminant of $\mathrm{A}$.

Proof. Let $A$ be $n \times n$ and let $r$ be the degree of the largest nonvanishing principal minor of $A$. As each $(r+t) \times(r+t)$ principal minor of $A$ is zero, and as the coefficient of $\lambda^{n-r-t}$ in the characteristic polynomial $f(\lambda)=\operatorname{det}\left(\lambda I_{n}-A\right)$ is (up to sign) the sum of all $(r+t) \times(r+t)$ principal minors of $A$, it follows that $f(\lambda)=\lambda^{n-r} g(\lambda)$. Thus $A$ has at most $r$ nonzero eigenvalues. Since for the type of matrix under discussion, the rank equals the number of nonzero eigenvalues, it follows that $r \geqslant$ $\operatorname{rank} A$. But clearly, from the determinantal characterization of $\operatorname{rank}, r \leqslant \operatorname{rank} A$. Hence $r=\operatorname{rank} A$.

The class considered in Theorem 4 includes, of course, all diagonable matrices, and hence all normal matrices. The example of the companion matrix of the polynomial $\lambda^{n}-1$ shows that for this class of matrices nothing can be asserted about the nonvanishing of the principal $t \times t$ minors, for $t<\operatorname{rank} A$. (In this example, all $t \times t$ principal minors are zero, for $t<n$.)

The class considered in the next theorem includes the Hermitian and the arbitrary symmetric matrices. For these matrices a fairly rich body of facts about principal submatrices is known. The discussion that follows is partly an expository summation of known results and partly a presentation of a number of new results.

THEOREM 5. Let $\mathfrak{F}$ be an arbitrary field with an automorphism $\sigma$, and let $\mathrm{A}$ be a matrix over is such that

$$
\left(\mathrm{A}^{\mathrm{T}}\right)^{\sigma}=\alpha \mathrm{A}
$$

where $\alpha \epsilon \mathfrak{\wp}$ and is nonzero. Then rank A equals the degree of the largest nonvanishing principal minor of $\mathbf{A}$.

Proof. Let $r=\operatorname{rank} A$ and let rows $i_{1}, i_{2}, \ldots, i_{r}$ of $A$ be linearly independent. From (4) it follows that columns $i_{1}, i_{2}, \ldots, i_{r}$ are also linearly independent. It is a known result, due to Marvin Marcus, that in a matrix of rank $r, r$ linearly independent rows and $r$ linearly independent columns meet in a nonsingular submatrix. In the present situation we see that $A\left[i_{1}, \ldots, i_{r} \mid i_{1}, \ldots, i_{r}\right]$ is a nonsingular principal submatrix. 
We now assume that automorphism $\sigma$ is involutory: $\sigma^{2}$ is the identity. In this case, it is easy to see that $\alpha \alpha^{\sigma}=1$ so that, if $\sigma$ is the identity, matrix $A$ is either symmetric or skew symmetric. If $\alpha=z^{\sigma} / z$ for some nonzero $z \epsilon \mathfrak{\curlyvee}$, then the multiple $B=z^{\sigma} A$ of $A$ is "Hermitian": $B^{\sigma T}=B$.

If $A_{1}$ and $A_{2}$ are principal submatrices of $A$, we say $A_{1}$ is nested in $A_{2}$, or contained in $A_{2}$, if $A_{1}$ is a (principal) submatrix of $A_{2}$. We write $A_{1} \subset A_{2}$. If rank $A=r$, a complete nested chain is a nested chain $A_{1} \subset A_{2} \subset \ldots \subset A_{r}$ of principal submatrices with degree $A_{1}=i$ for $i=1,2, \ldots, r$, such that in the sequence

$$
\text { 1, } \operatorname{det} A_{1}, \operatorname{det} A_{2}, \ldots, \operatorname{det} \mathrm{A}_{r-1}, \operatorname{det} A_{r},
$$

$\operatorname{det} A_{r} \neq 0$ and no two consecutive terms are zero.

Theorem 6. Assume the hypotheses of Theorem 5 and let $\sigma$ be involutory. Let rank $\mathrm{A}=\mathrm{r}$. Let $\mathrm{B}_{\mathrm{i}} \subset \mathrm{B}_{\mathrm{j}} \subset \ldots \subset \mathrm{B}_{\mathrm{p}} \subset \mathrm{B}_{\mathrm{q}}$ be a nested set of nonsingular principal submatrices of $\mathrm{A}$, of degrees $\mathrm{i}, \mathrm{j}, \ldots ., \mathrm{p}, \mathrm{q}$ respectively. Then it is possible to find a complete nested chain $\mathrm{A}_{1} \subset \ldots$ that $\mathrm{A}_{\mathrm{i}}=\mathrm{B}_{\mathrm{i}}, \mathrm{A}_{\mathrm{j}}=\mathrm{B}_{\mathrm{j}}, \ldots ., \mathrm{A}_{\mathrm{p}}=\mathrm{B}_{\mathrm{p}}, \mathrm{A}_{\mathrm{q}}=\mathrm{B}_{\mathrm{q}}$.

Proof. First note that each principal submatrix of $A$ will also satisfy (4). Observe also that $\left(C^{T \sigma} A C\right)^{T \sigma}=\alpha C^{T \sigma} A C$ is equivalent to (4), for any nonsingular $C$. Because of this last fact we may perform elementary column operations (and the corresponding row operations) on $A$ to obtain a new matrix satisfying (4).

Let $M$ be a nonsingular principal $m$-square submatrix of $A$, with $m<r$. Without affecting any inclusion relation among the principal submatrices of $A$, we may perform a permutation similarity of $A$ to put $M$ into the upper left corner of $A$ :

$$
A=\left[\begin{array}{ll}
M & A_{12} \\
A_{21} & A_{22}
\end{array}\right] .
$$

As the matrix $\left[M, A_{12}\right]$ is $m \times n$ and has rank $m$, each column of $A_{12}$ is a linear combination of the columns of $M$. By adding appropriate linear combinations of the columns of $A$ passing through $M$ to the columns of $A$ passing through $A_{12}$, we may replace $A_{12}$ with a zero matrix. Perform also the corresponding row operations. Each principal submatrix of $A$ containing $M$ has its determinant unaffected by these elementary operations. So we pass to $\widetilde{A}=\operatorname{diag}\left(M, \widetilde{A}_{22}\right)$. We claim that $\widetilde{A}$ has either a nonsingular $(m+1)$-square principal submatrix containing $M$ or a nonsingular $(m+2)$ square principal submatrix containing $M$. If this statement were false, each principal $1 \times 1$ and each principal $2 \times 2$ submatrix of $\widetilde{A}_{22}$ would be singular. An elementary calculation shows (using (4)) that $\widetilde{A_{22}}=0$ would result, contradicting the fact that $\operatorname{rank} \widetilde{A}>m$. Returning to the original $A$, we have proved that if $M$ is a nonsingular $m$-square principal submatrix of $A$ with $m<\operatorname{rank} A$, then $M$ is contained in a nonsingular $t$-square principal submatrix of $A$, where $t$ is either $m+1$ or $m+2$.

We use the result of the last paragraph to build a chain from $B_{i}$ to $B_{j}$. Let $A_{i}=B_{i}=M$ and apply the result just proved to $A_{j}=B_{j}$. Either, within $A_{j}, A_{i}$ is nested in a nonsingular $(i+1)$-square principal submatrix, call it $A_{i+1}$, or $A_{i}$ is nested in a nonsingular $(i+2)$-square principal submatrix, call it $A_{i+2}$. In the latter case let $A_{i+1}$ be any principal submatrix nested between $A_{i}$ and $A_{i+2}$. Repeating with $M=A_{i+1}$ or $M=A_{i+2}$, we build a chain within $B_{j}$. The same method, applied to $A$, can be used to build a chain from $A_{q}=B_{q}$ up to some $A_{r}$ with det $A_{r} \neq 0$. To build a chain up to $B_{i}$, we start by noting that $B_{i}$ must contain a nonsingular principal $1 \times 1$ or $2 \times 2$ submatrix, since otherwise we would get $B_{i}=0$. Combining all of these chains yields the required complete nested chain within $A$.

That we cannot avoid the presence of zero terms in (5) is shown clearly by the next theorem.

THeOREм 7. Assume the hypotheses of Theorems 5 and 6 . Suppose also that $\alpha=\mathrm{z}^{\sigma} / \mathrm{z}$ is not true for any nonzero z $\epsilon \mathfrak{r}$. Then each principal minor of $\mathrm{A}$ of odd degree is zero. Furthermore, the rank of $\mathrm{A}=2 \mathrm{~s}$ is an even integer and $\mathrm{A}$ has a nonzero $2 \mathrm{t}$-square principal minor for each $\mathrm{t}=1,2, \ldots, \mathrm{s}$.

Proof. From (4) the main diagonal elements $a_{i i}$ of $A$ satisfy $a_{i i}^{\sigma}=\alpha a_{i i}$. Hence each $a_{i i}=0$. If $M_{1}$ is a nonsingular $t$-square principal minor of $A$ and $M_{2}$ is a nonsingular $(t+1)$-square principal minor of $A$, then from (4) we get $\left(\operatorname{det} M_{1}\right)^{\sigma}=\alpha^{t} \operatorname{det} M_{1}$ and $\left(\operatorname{det} M_{2}\right)^{\sigma}=\alpha^{t+1} \operatorname{det} M_{2}$. Thus $\alpha=z^{\sigma} / z$ 
with $z=\operatorname{det} M_{2} / \operatorname{det} M_{1}$. This is a contradiction and hence at least one of $\operatorname{det} M_{1}$, det $M_{2}$ is zero. Thus in any complete chain, every term $\operatorname{det} A_{i}$ in (5) with $i$ odd must be zero and every term $\operatorname{det} A_{i}$ with $i$ even must be nonzero. This completes the proof.

The result of Theorem 7 includes, as a special case, a known result for skew symmetric matrices.

Theorem 8. Assume the hypotheses of Theorems 5 and 6. Suppose that A has exactly one nonsingular principal $\mathrm{k}$-square submatrix, call it $\mathrm{M}$, and that each principal $(\mathrm{k}+1)$-square submatrix is singular. Then $\mathrm{k}=$ rank $\mathrm{A}$ and

$$
A=P\left[\begin{array}{ll}
M & 0 \\
0 & 0
\end{array}\right] P^{T}
$$

where $\mathrm{P}$ is a permutation matrix.

Proof. After a permutation similarity of $A$, we may move $M$ to the upper left corner of $A$, and so we may assume that $A$ is given by (6). Let $a$ be any main diagonal element of $A_{22}$. By Theorem 6 , if $a \neq 0$, we may find a complete nested chain passing through (a). Such a nested chain cannot have $M$ as its degree $k$ member since $a \notin M$, so the degree $k$ member in this chain is singular. If $k=r$ we have a contradiction. If $k<r$, the chain containing (a) cannot have any degree $k+1$ nonsingular member either, since any such principal submatrix of $A$ is singular. Thus $a=0$ for each diagonal element $a$ of $A_{22}$. Let

$$
\left[\begin{array}{ll}
\beta & b \\
c & 0
\end{array}\right], b, c \notin M,
$$

be any principal $2 \times 2$ submatrix of $A$ not contained within $M$. By the same argument, if $b c \neq 0$, we would get a contradiction, provided $k \neq 1$. So $b=c=0$. This is also true if $k=1$. Thus $A_{22}=0$ and all elements of $A_{12}$ and $A_{21}$ are zero, so that $A_{12}=0$ and $A_{21}=0$. This completes the proof.

It is of interest to see the analogue of Theorem 8 for arbitrary matrices.

Theorem 9. Let $\mathrm{A}$ be an arbitrary $\mathrm{m} \times \mathrm{n}$ matrix over field $\mathfrak{\wp}$. Suppose that $\mathrm{A}$ has one, and only one, nonsingular $\mathrm{k} \times \mathrm{k}$ submatrix $\mathrm{M}$. Then $\mathrm{k}=\operatorname{rank} \mathrm{A}$ and

$$
A=P\left[\begin{array}{ll}
M & 0 \\
0 & 0
\end{array}\right] Q
$$

where $\mathrm{P}$ and $\mathrm{Q}$ are permutation matrices.

Proof. After passage from $A$ to $P A Q$, we may assume that $A$ is given by (6). Let $M_{1}$ be the $k \times k$ submatrix of $A$ obtained by using columns $1, . \bullet^{\circ}, i-1, i+1, \ldots, k$ of $M$ and one column of $A_{12}$. This matrix is singular. As the columns of $M$ used are linearly independent, we see that a given column of $A_{12}$ is a linear combination of columns $1, \ldots, i-1, i+1, \ldots, k$ of $M$. This is true for each $i=1,2, \ldots, k$, from which it is not difficult to see that the given column of $A_{12}$ is zero. Thus $A_{12}=0$ and similarly $A_{21}=0$. The $k \times(k-1)$ submatrix of $M$ obtained by using the first $k-1$ columns of $M$ has rank $k-1$, and hence must have a $(k-1)$-square nonsingular submatrix $\widetilde{M}$. If $i>k, j>k$ then

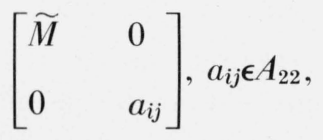

is a $k \times k$ submatrix of $A$ other than $M$ and hence is singular. So $a_{i j}=0$ for all $a_{i j} \in A_{22}$. Thus $A_{22}=0$. This completes the proof.

We now assume that we are dealing with complex Hermitian or real symmetric matrices. In this case we have the following known fact (Gundelfinger's rule [6]). Our proof is new. 
THEOREM 10. Let A be a Hermitian or real symmetric matrix with rank $\mathrm{A}=\mathrm{r}$. In any complete nested chain $\mathrm{A}_{1} \subset \mathrm{A}_{2} \subset \ldots \subset \mathrm{A}_{\mathrm{r}}$, the number of permanences of sign in (5) equals the number of positive eigenvalues of $\mathrm{A}$, and the number of variations of sign equals the number of negative eigenvalues of A. Either sign may be attached to a zero term in (5).

Proof. Recall the fact that the eigenvalues of a principal $(k-1)$-square submatrix of a $k$-square Hermitian matrix $H$ interlace the eigenvalues of $H$. For a very short proof of this fact, see [2]. We use this fact to show by induction on $i$ that in a complete nested chain $A_{1} \subset \ldots \subset A_{r}$, if $\operatorname{det} A_{i} \neq 0$, the number of positive (negative) eigenvalues of $A_{i}$ equals the number $p_{i}\left(v_{i}\right)$ of permanences (variations) of sign in the sequence $1, \operatorname{det} A_{1}, \ldots, \operatorname{det} A_{i}$. There are two cases. If det $A_{i-1} \neq 0$ then $A_{i-1}$ has $p_{i-1}$ positive and $v_{i-1}$ negative eigenvalues. By interlacing, $A_{i}$ has $p_{i-1}$ positive and $v_{i-1}$ negative eigenvalues, and one eigenvalue $\lambda$ which may be positive or negative. If $\lambda$ is positive (negative) it follows (since the determinant is the product of the eigenvalues) that $\operatorname{det} A_{i}$ and $\operatorname{det} A_{i-1}$ have the same (opposite) signs. Thus $A_{i}$ has $p_{i}$ positive and $v_{i}$ negative eigenvalues. If $\operatorname{det} A_{i-1}=0$ then $\operatorname{det} A_{i-2} \neq 0$, and $A_{i-2}$ has $p_{i-2}\left(v_{i-2}\right)$ positive (negative) eigenvalues. By interlacing and det $A_{i-1}=0, A_{i-1}$ has $p_{i-2}$ positive, $v_{i-2}$ negative and one zero eigenvalue. By interlacing and det $A_{i} \neq 0, A_{i}$ has $p_{i-2}+1$ positive and $v_{i-2}+1$ negative eigenvalues. Thus $\operatorname{det} A_{i}$ and det $A_{i-2}$ have opposite signs, so that $p_{i}=p_{i-2}+1\left(v_{i}=v_{i-2}+1\right)$ for any choice of the sign of det $A_{i-1}$, and moreover there always is one permanence and one variation in the sequence $\operatorname{det} A_{i-2}$, $\operatorname{det} A_{i-1}$, det $A_{i}$. The proof is now completed by noting that $p_{r}+v_{r}=r$ is the number of nonzero eigenvalues of $A$ (since $\operatorname{rank} A=r$ ) and that, by interlacing, if $A_{r}$ has $p_{r}$ positive and $v_{r}$ negative eigenvalues, then $A$ has at least $p_{r}$ positive and $v_{r}$ negative eigenvalues.

An arbitrary nested set $A_{1} \subset \ldots \subset \mathrm{A}_{r}$ with det $A_{r} \neq 0$ does not necessarily qualify for use in Theorem 10, since in (5), there may be two or more consecutive zero terms. It was noted by Frobenius [7] that these nested chains may still be used in Theorem 10, provided that not too many zeros are present. We give an improvement of the result of Frobenius, with a new proof.

Theorem 11. Let rank $\mathrm{A}=\mathrm{r}$ and let $\mathrm{A}_{1} \subset \ldots \subset \mathrm{A}_{\mathrm{r}}$ be a nested chain with det $\mathrm{A}_{\mathrm{r}} \neq 0$. Suppose, in (5), there are never more than three consecutive zeros. Then the conclusion of Theorem 10 is valid provided:

(i) whenever two consecutive zeros appear, say

$$
\operatorname{det} A_{i-3} \neq 0, \operatorname{det} A_{i-2}=0, \operatorname{det} A_{i-1}=0, \operatorname{det} A_{i} \neq 0 \text {, }
$$

we assign to det $\mathrm{A}_{\mathrm{i}-2}$ and det $\mathrm{A}_{\mathrm{i}-1}$ the signs of $-\operatorname{det} \mathrm{A}_{\mathrm{i}-3}$;

(ii) whenever three consecutive zeros appear, say

$$
\operatorname{det} A_{i-4} \neq 0, \operatorname{det} A_{i-3}=0, \operatorname{det} A_{i-2}=0, \operatorname{det} A_{i-1}=0, \operatorname{det} A_{i} \neq 0,
$$

always det $\mathrm{A}_{\mathrm{i}-4}$ and det $\mathrm{A}_{\mathrm{i}}$ have the same sign, and we assign to det $\mathrm{A}_{\mathrm{i}-3}$, det $\mathrm{A}_{\mathrm{i}-2}$, det $\mathrm{A}_{\mathrm{i}-1}$ the sign of - det $\mathrm{A}_{\mathrm{i}-4}$.

Proof. We prove the inductive assertion of the previous proof. First consider the case of two consecutive zeros (7). Suppose $A_{i-3}$ has $p_{i-3}\left(v_{i-3}\right)$ positive (negative) eigenvalues. By interlacing, $A_{i-2}$ has $p_{i-3}$ positive, one zero, and $v_{i-3}$ negative eigenvalues. If $A_{i-1}$ had zero as a double root, interlacing would force $A_{i}$ to have a zero eigenvalue, a contradiction. By interlacing we therefore see that $A_{i-1}$ has either $p_{i-3}+1$ positive, one zero, and $v_{i-3}$ negative eigenvalues, or else $p_{i-3}$ positive, one zero, and $v_{i-3}+1$ negative eigenvalues. In the first case $A_{i}$ has, by interlacing, $p_{i-3}+2$ positive and $v_{i-3}+1$ negative eigenvalues (so that $\operatorname{det} A_{i}=-\operatorname{det} A_{i-3}$ ). In the second case $A_{i}$ has $p_{i-3}+1$ positive and $v_{i-3}+2$ negative eigenvalues (so that $\operatorname{det} A_{i}=\operatorname{det} A_{i-3}$ ). By counting the permanences and variations in (7), we now establish the inductive step.

Next consider the case (8) of three consecutive zeros. By interlacing, we verify in the same way if $A_{i-4}$ has $p_{i-4}$ positive $\left(v_{i-4}\right.$ negative) eigenvalues, then $A_{i}$ has $p_{i-4}+2$ positive $\left(v_{i-4}+2\right.$ negative) eigenvalues. Moreover, in (8), there are exactly two permanences and two variations of sign.

The proof is now completed as in the previous theorem.

We note that Theorem 10 can be used to prove the law of inertia. 
Theorem 12. Let A and $\mathrm{B}=\mathrm{C}^{*} \mathrm{AC}$ be conjunctive Hermitian matrices. Then $\mathrm{B}$ and $\mathrm{A}$ have the same number of positive eigenvalues and the same number of negative eigenvalues.

Proof. Let $C=U D V$ where $U$ and $V$ are unitary and $D=\operatorname{diag}\left(d_{1}, \ldots, d_{n}\right)$. The existence of this factorization is an immediate consequence of the polar factorization theorem. Then

$$
B=V^{-1} D\left(U^{-1} A U\right) D V=V^{-1} \mathfrak{B} V
$$

where $\mathfrak{B}=D a D$ and $a=U^{-1} A U$. It suffices to prove the result for $\mathfrak{B}$ and $a$. If

$$
A_{k}=\mathfrak{a}\left[i_{1}, \ldots, i_{k} \mid i_{1}, \ldots, i_{k}\right]
$$

then det $\mathfrak{B}\left[i_{1}, \ldots, i_{k} \mid i_{1}, \ldots, i_{k}\right]=\left(d_{i_{1}} \ldots d_{i_{k}}\right)^{2}$ det $\mathfrak{a}\left[i_{1}, \ldots, i_{k} \mid i_{1}, \ldots, i_{k}\right]$. From this fact, it follows that from a complete nested chain for $\mathfrak{a}$, a complete nested chain for $\mathfrak{B}$ can be constructed using the same sets of rows and columns, such that the sign patterns in the chains for $\mathfrak{a}$ and $\mathfrak{B}$ are the same. An appeal to Theorem 10 completes the proof.

\section{Symmetric Matrices With Sets of Equal Principal Minors}

In this section we study symmetric matrices $\mathrm{A}$, over an arbitrary field $\mathfrak{F}$, for which:

(i) all $r \times r$ principal minors of $A$ are equal;

(ii) all $(r+1) \times(r+1)$ principal minors of $A$ are equal;

(iii) all $(r+2) \times(r+2)$ principal minors of $A$ are equal;

(iv) $\operatorname{rank} A \geqslant r+3$.

Any matrix $A$ of the form

$$
A=D\left(a I_{n}+b J_{n}\right) D^{-1}, \quad a, b \epsilon \mathfrak{F},
$$

where $D$ is a diagonal matrix of plus and minus ones, will be symmetric and will have the property

(v) all $t \times t$ principal minors are equal,

for each $\mathrm{t}=1,2, \ldots, n$. In this section we prove the following theorem.

Theorem 13. Let $\mathbf{n} \times \mathbf{n}$ symmetric A satisfy (i), (ii), (iii), and also (iv) if $\mathrm{r}>1$. Then $\mathbf{A}$ is given by (9), and hence A satisfies (v) for each $\mathrm{t}=1,2, \ldots, \mathrm{n}$.

Proof.

CAsE 1. Let $r=1$. Then, by (i), all diagonal elements of $A$ are equal. Using this and (ii), we see that $a_{i j}^{2}=\alpha^{2}$ for $i<j$, where $\alpha \epsilon \mathfrak{F}$. So $A=a I_{n}+\alpha E$, where $E=\left(\epsilon_{i j}\right)$ is such that $\epsilon_{i i}=1$ for each $i$ and $\epsilon_{i j}= \pm 1$ for $i \neq j$. If $\mathfrak{\&}$ has characteristic two we have proved that $A$ is given by (9). Suppose the characteristic of $\mathfrak{F}$ is not two. If we pass to $D A D^{-1}$ where $D=\operatorname{diag}\left(1, \epsilon_{12}, \epsilon_{13}, \ldots ., \epsilon_{1 n}\right)$, we reduce the situation to the case in which $\epsilon_{12}=\epsilon_{13}=\ldots .=\epsilon_{1 n}=1$. By property (iii), we compute that $2 \epsilon_{i j} \epsilon_{j k} \epsilon_{i k}=2 \beta$ is independent of $i, j, k$ where $i<j<k$. Taking $i=1$, we get $\epsilon_{j k}=\beta$ for all $i<j<k$. Let $D=\operatorname{diag}(\beta, 1,1, \ldots, 1)$. Then $D A D^{-1}=a I_{n}+\alpha \beta J_{n}$. This completes the proof in Case 1 .

CASE 2. Let $r=n-3$. This is the maximum possible value of $r$ and, by (iv), implies that $A$ is nonsingular. Let $C_{s}(A)$ denote the $s$ th compound of $A$ and let $C^{n-s}(A)$ denote the sth supplementary compound. For the definitions and properties of these matrices, see [5]. For our purposes, the important fact about $C_{s}(A)$ is that its main diagonal consists of all $s \times s$ principal minors of $A$, and the important fact about $C^{n-s}(A)$ is that its main diagonal consists of all $(n-s) \times(n-s)$ principal minors of $A$. Moreover we have

$$
C_{s}(\operatorname{adj} A)=(\operatorname{det} A)^{s-1} C^{n-s}\left(A^{T}\right) .
$$

Here adj $A$ is the adjugate of $A$. As $A$ is symmetric we may drop T. Also adj $A$ is symmetric. 
It follows from (10), with $s=1,2,3$, that adj $A$ has all $s \times s$ principal minors equal. Thus adj $A$ falls into Case 1 , and hence

$$
\text { adj } A=D\left(a^{\prime} I_{n}+b^{\prime} J_{n}\right) D^{-1}, \quad a^{\prime}, b^{\prime} \epsilon \mathfrak{r} .
$$

However, $A^{-1}=(\operatorname{det} A)^{-1}$ adj $A$. Since $A$ is a polynomial in $A^{-1}$, it follows that $A=D_{p}\left(J_{n}\right) D^{-1}$ for some polynomial $p(\lambda)$. Since $J_{n}^{2}=n J_{n}$, any polynomial in $J_{n}$ equals a linear polynomial in $J_{n}$. Therefore $A$ is given by (9).

General case. This case is a chase through the submatrices of $A$, using Theorem 6 and repeated reductions to the circumstances of Cases 1 or 2 . We may assume that $2 \leqslant r \leqslant n-4$ since Cases 1 and 2 disposed of the situations $r=1$ and $r=n-3$.

Our first object is to prove that any $r \times r$ principal submatrix $A_{r}$ of $A$ is of the form

$$
A_{r}=D_{r}\left(a_{r} I_{r}+b_{r} J_{r}\right) D_{r}^{-1}, \quad a_{r}, b_{r} \in \widetilde{F}
$$

with $D_{r}$ a diagonal matrix of plus or minus ones. If $A_{r}$ is nested in a nonsingular principal $(r+3) \times(r+3)$ submatrix $A_{r+3}$, then an application of Case 2 to $A_{r+3}$ shows that

$$
A_{r+3}=D_{r+3}\left(a_{r+3} I_{r+3}+b_{r+3} J_{r+3}\right) D_{r+3}^{-1},
$$

and hence $A_{r}$ has the form (11).

If $\operatorname{det} A_{r} \neq 0$, we can pass a complete nested chain through $A_{r}$ and so we see that either $A_{r}$ is contained in a nonsingular $(r+3)$-square principal submatrix $A_{r+3}$, or else $A_{r}$ is contained in a nonsingular $(r+4)$-square principal submatrix $A_{r+4}$. If $\operatorname{det} A_{r}=0$, then by (i) every $r \times r$ principal minor of $A$ is zero. Thus any complete nested chain must pass through a nonsingular $(r+1)$-square principal submatrix. By (ii), every $(r+1)$-square principal minor is therefore nonzero. Let $A_{r+1}$ be any $(r+1)$-square principal submatrix containing $A_{r}$. Thus $A_{r+1}$ is nonsingular. Any complete nested chain passing through $A_{r+1}$ must either pass through a nonsingular $(r+3)$-square principal submatrix $A_{r+3}$ or a nonsingular $(r+4)$-square principal submatrix $A_{r+4}$. Thus we see that always $A_{r} \subset A_{r+3}$ where $A_{r+3}$ is nonsingular, or $A_{r} \subset A_{r+4}$ where $A_{r+4}$ is nonsingular.

If $A_{r} \subset A_{r+3}$ with $A_{r+3}$ nonsingular, we have achieved our object as indicated in a paragraph above.

So let $A_{r} \subset A_{r+4}$ where $A_{r+4}$ is nonsingular. To prove that $A_{r}$ is of the form (11), it suffices to prove that $A_{r+4}$ is of this form. Of course $A_{r+4}$ has the property that all $t \times t$ principal minors are equal, for $t=r, r+1, r+2$. Now

$$
C_{s}\left(\operatorname{adj} A_{r+4}\right)=\left(\operatorname{det} A_{r+4}\right)^{s-1} C^{r+4-s}\left(A_{r+4}\right) .
$$

For $s=2,3,4$, formula (12) implies that in $B=\operatorname{adj} A_{r+4}$, all principal $t \times t$ minors are equal, for $t=2,3,4$. We will prove that all $1 \times 1$ principal minors of $B$ are equal. If we can do this, an application of Case 1 will then show that $B$ has the form (11), and the argument at the end of Case 2 will then show that $A_{r+4}$ has the form (11).

Since $r \geqslant 2, B$ is at least $6 \times 6$, and furthermore, $B$ is nonsingular. To prove that all $1 \times 1$ principal minors of $B$ are equal, it will be enough to prove that the two main diagonal elements of any $2 \times 2$ principal submatrix of $B$ are equal. Let $B$. be a principal $2 \times 2$ submatrix of $B$. Since all $t \times t$ principal minors of $B$ are equal, for $t=2,3,4$, an argument used above now shows that either $B_{2} \subset B_{5}$ or $B_{2} \subset B_{6}$, where $B_{5}$ is a nonsingular $5 \times 5$ principal submatrix of $B$, or $B_{6}$ is a nonsingular $6 \times 6$ principal submatrix of $B$. If $B_{2} \subset B_{5}$, then $B_{5}$ is nonsingular and has all $t \times t$ principal minors equal, for $t=2,3,4$. An application of Case 2 to $B_{5}$ shows that $B_{5}$ has the form (11). Hence the main diagonal of $B_{5}$, and therefore of $B_{2}$, is constant. If $B_{2} \subset B_{6}$, then either all principal $5 \times 5$ minors of $B_{6}$ are zero, or there is at least one not zero. In the first case, $B_{6}$ has all $t \times t$ principal minors equal for $t=3,4,5$, and hence by Case $2, B_{6}$ has the form (11), and so the main diagonal 
of $B_{6}$ (and therefore of $B_{2}$ ) is constant. So we may suppose that at least one prinicpal $5 \times 5$ submatrix, say $B_{5}^{\prime}$, of $B_{6}$ is nonsingular. If $B_{2} \subset B_{5}^{\prime}$, we are reduced to the earlier case of $B_{2} \subset B_{5}$. So $B_{2} \nsubseteq B_{5}^{\prime}$. Since $B_{5}^{\prime}$ has all $t \times t$ principal minors equal for $t=2,3,4$, an application of Case 2 to $B_{5}^{\prime}$ shows that $B_{5}^{\prime}=D_{5}^{\prime}\left(a I_{5}+b J_{5}\right) D_{5}^{\prime-1}$. Without loss of generality we may suppose that $B_{2}$ is in the upper left corner of $B_{6}$ and that $B_{5}^{\prime}$ is in the lower right corner of $B_{6}$. Passing from $B_{6}$ to

$$
\operatorname{diag}\left(1, D_{5}^{\prime}\right) B_{6} \operatorname{diag}\left(1, D_{5}^{\prime}\right)^{-1} \text {, }
$$

we may now assume that $B_{5}^{\prime}=a I_{5}+b J_{5}$. So we have

$$
B_{6}=\left[\begin{array}{llllll}
x & c_{2} & c_{3} & c_{4} & c_{5} & c_{6} \\
c_{2} & a+b & b & b & b & b \\
c_{3} & b & a+b & b & b & b \\
c_{4} & b & b & a+b & b & b \\
c_{5} & b & b & b & a+b & b \\
c_{6} & b & b & b & b & a+b
\end{array}\right]
$$

The lower right $5 \times 5$ submatrix is $B_{5}^{\prime}$, and

$$
B_{2}=\left[\begin{array}{ll}
x & c_{2} \\
c_{2} & a+b
\end{array}\right]
$$

We must prove that $x=a+b$. Note that $a \neq 0$ since $B_{5}^{\prime}$ is nonsingular.

The equality of the principal $2 \times 2$ minors of $B_{6}$ gives

$$
x(a+b)-c_{i}^{2}=\operatorname{det}\left[\begin{array}{ll}
a+b & b \\
b & a+b
\end{array}\right] .
$$

Thus $c_{i}^{2}$ is independent of $i$. So let $c_{i}=\epsilon_{i} c$, where $\epsilon_{i}= \pm 1$. We want to prove that $\epsilon_{2}=\epsilon_{3}=\epsilon_{4}=\epsilon_{5}=\epsilon_{6}$. If $\widetilde{s}$ has characteristic two this is immediate. If $c=0$ we can choose this to be so. If $c \neq 0$ but $b=0$, we can make $\epsilon_{2}=\epsilon_{3}=\epsilon_{4}=\epsilon_{5}=\epsilon_{6}$ by passing to

$$
\operatorname{diag}\left(1, \epsilon_{2}, \epsilon_{3}, \epsilon_{4}, \epsilon_{5}, \epsilon_{6}\right) B_{6} \operatorname{diag}\left(1, \epsilon_{2}, \epsilon_{3}, \epsilon_{4}, \epsilon_{5}, \epsilon_{6}\right)^{-1}
$$

So to prove that $\epsilon_{2}=\epsilon_{3}=\epsilon_{4}=\epsilon_{5}=\epsilon_{6}$, we may assume $b c \neq 0$ and that the characteristic of $\mathfrak{\mho}$ is not two. The equality of the principal $3 \times 3$ minors of $B_{6}$ gives

$$
x(a+b)^{2}+2 b c^{2} \epsilon_{i} \epsilon_{j}-2(a+b) c^{2}-b^{2} x=\operatorname{det}\left[\begin{array}{lll}
a+b & b & b \\
b & a+b & b \\
b & b & a+b
\end{array}\right], \quad i<j .
$$

Thus $\epsilon_{i} \epsilon_{j}$ is independent of $i$ and $j$ for $i<j$. Taking $i=2$, we get $\epsilon_{3}=\epsilon_{4}=\epsilon_{5}=\epsilon_{6}$. Then $\epsilon_{2} \epsilon_{3}=\epsilon_{3} \epsilon_{4}$, 
so that $\epsilon_{2}=\epsilon_{4}$. Thus we have $\epsilon_{2}=\epsilon_{3}=\epsilon_{4}=\epsilon_{5}={ }_{6}=\epsilon$. Changing $\epsilon c$ to $c$, we absorb $\epsilon$ and now

$$
B_{6}=\left[\begin{array}{llllll}
x & c & c & c & c & c \\
c & a+b & b & b & b & b \\
c & b & a+b & b & b & b \\
c & b & b & a+b & b & b \\
c & b & b & b & a+b & b \\
c & b & b & b & b & a+b
\end{array}\right] .
$$

The equality of the principal $2 \times 2$ minors and of the principal $3 \times 3$ minors of $B_{6}$ gives

$$
\operatorname{det}\left[\begin{array}{ll}
x & c \\
c & a+b
\end{array}\right]=\operatorname{det}\left[\begin{array}{ll}
a+b & b \\
b & a+b
\end{array}\right],
$$

and

$$
\operatorname{det}\left[\begin{array}{lll}
x & c & c \\
c & a+b & b \\
c & b & a+b
\end{array}\right]=\operatorname{det}\left[\begin{array}{lll}
a+b & b & b \\
b & a+b & b \\
b & b & a+b
\end{array}\right] .
$$

By expanding the left sides of (13) and (14), we see that (13) and (14) are a set of two nonhomogeneous linear equations in unknowns $x$ and $c^{2}$. The coefficient determinant of $x$ and $c^{2}$ is

$$
\operatorname{det}\left[\begin{array}{ll}
a+b & -1 \\
a^{2}+2 a b & -2 a
\end{array}\right]=-a^{2} .
$$

Since $a \neq 0$, system (13)-(14) has a unique solution for unknowns $x$ and $c^{2}$. An obvious solution is $x=a+b$ and $c^{2}=b^{2}$. Therefore, we have finally succeeded in proving that $B_{2}$ has constant main diagonal.

We have demonstrated that each $A_{r} \subset A$ is of the form (11). Hence each $A_{r}$ has the property: all $t \times t$ principal minors of $A_{r}$ are equal, for $t=1,2, \ldots, r-1$. Let $A_{r}$ and $\bar{A}_{r}$ be any two $r \times r$ principal submatrices of $A$. We may connect $A_{r}$ and $\bar{A}_{r}$ with a chain of $r \times r$ principal submatrices of $A$,

$$
A_{r}, A_{r}^{\prime}, A_{r}^{\prime \prime}, \ldots, \bar{A}_{r}
$$

such that each principal submatrix in this chain overlaps the next in $r-1$ main diagonal positions. For $t \leqslant r-1$, all the principal $t \times t$ submatrices contained in one matrix in this chain have the same determinant as all the principal $t \times t$ submatrices contained in the next matrix in the chain. This is so since there is a $t \times t$ principal submatrix common to these adjacent matrices in the chain. It therefore follows that all $t \times t$ principal minors of $A$ are equal; $t=1,2, \ldots ., r-1$. Therefore all $t \times t$ principal minors of $A$ are equal, for $t=1,2, \ldots, r+3$. Therefore all $t \times t$ principal minors of $A$ are equal, for $t=1,2,3$. We may now apply Case 1 to show that $A$ has the form (9). The proof is now complete.

If rank $A<r$ it is, of course, true that $A$ satisfies (i), (ii), and (iii) in a trivial way. In this situation we should not expect to deduce anything about $A$. It is an open question whether any conclusion can be drawn if $A$ satisfies (i), (ii), (iii), if $\operatorname{rank} A$ is $r, r+1$, or $r+2$, and if degree $A \geqslant r+3$.

There appears to be no simple counterpart of Theorem 13 for Hermitian matrices. 


\section{References}

[1] R. C. Thompson, Principal submatrices of normal and Hermitian matrices, Illinois J. Math. 10, 296-308 (1966).

[2] R. C. Thompson and P. McEnteggert, Principal submatrices II: The upper and lower quadratic inequalities, to appear in J. Linear Algebra and Appl.

[3] R. C. Thompson, Principal submatrices III: Linear inequalities, J. Res. NBS 72B (Math. Sci.), No. 1, 7 (1968).

[4] - Principal submatrices IV: On the independence of the eigenvalues of different principal submatrices, submitted to J. Linear Algebra and Appl.

[5] J. H. M. Wedderburn, Lectures on Matrices, Amer. Math. Soc. Colloq. Publ. New York, 1 7, 63-67 (1934).

[6] S. Gundelfinger, J. Reine angew. Math. 91, 221-237 (1881).

[7] G. Frobenius, Über das Tragheitsgesetz der quadratischen Formen, S. B. Deutsch Akad. Wiss. Berlin, Math-Nat-Kl, 24l-256, 407-431 (1894).

(Paper 72B2-265) 